\title{
Le grand dilemme en santé publique
}

\section{Eberhard Wolff}

Prof. Dr rer. soc., rédacteur culture, histoire, société

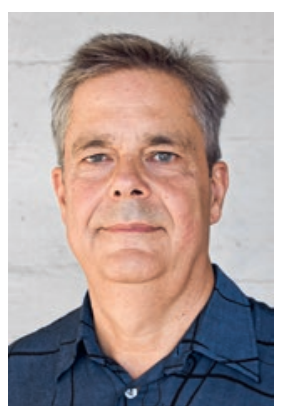

Il ne sera pas question d'internements d'office ni du recours aux forces de l'ordre dans le contexte de la crise du Covid-19. Du moins pas directement. System einer vollständigen medicinischen Polizey est le titre d'une compilation en six tomes, où le médecin allemand Johann Peter Frank (1745-1821) rassemblait ses idées en matière de santé publique. «Polizey» revêtait jadis le sens de police et de politique, mais quelle que soit l'interprétation, il était avant tout question dans l'ouvrage du «système de santé étatique». Johann Peter Frank est considéré aujourd'hui, de manière un peu réductrice, comme le fondateur de la santé publique. On reparle à nouveau de lui, le bicentenaire de sa mort ayant été célébré en mars dernier. Et l'ambiguïté évoquée plus haut est révélatrice du dilemme qui, aujourd'hui comme hier, caractérise les choix en matière de santé publique: doit-on guider ou imposer?

L'idée de la santé comme mission de l'Etat s'inscrit dans l'époque de Johann Peter Frank, l'absolutisme éclairé. On considérait alors qu'une population abondante et en bonne santé constituait la base indispensable d'un Etat fort, où les médecins étaient soumis à cet idéal de la santé. J. P. Frank était le tenant d'une médecine sociale dans laquelle la misère du peuple était à l'origine de toutes les maladies. Son "système» appelait à la mise en place de conditions de vie saines et à une bonne formation médicale, mais comportait aussi l'autre facette de «l'hygiène sociale», à savoir le contrôle et l'interventionnisme. Il a bâti un imaginaire rempli de craintes d'un «déclin sanitaire»: pour préserver la santé du peuple, il voulait voir le comportement et la vie intime de ce dernier réglés et ordonnés jusque dans le moindre détail, quitte à édicter des interdictions. Il condamnait les «beuveries» lors de célébrations familiales et incitait à la délation. Il voulait même interdire la valse aux «fêtes de village où le peuple s'égaillait». Mais surtout, refuser le droit de se marier à ceux qui souffraient de cachexie, d'émaciation, de goutte ou d'autres maux, mais aussi s'ils étaient vieux ou "mal faits». Il allait jusqu'à envisager des "interdictions de coït» ou des divorces forcés, tout cela dans une inextricable pelote mêlant le proto-eugénisme et la morale bourgeoise, convaincu de détenir l'unique vérité médicale comme l'exclusivité de la morale.
Si sa vision rencontra un large écho, des contemporains condamnèrent ses idées, les qualifiant de despotiques et de velléités dictatoriales. Les historiens de la médecine nazie allaient, eux, l'élever au rang de «Führer de la santé» et louer sa "politique raciale».

On ne s'étonnera pas que des historiens anglo-américains de la médecine aient pu décrire après-guerre la pensée de Frank comme relevant d'un «despotisme éclairé». Ils avaient décelé le dilemme fondamental à la base de toute politique de "santé publique»: les stratégies de prévention peuvent rapidement basculer dans la réglementation excessive et l'interventionnisme. Il a fallu plus de temps aux historiens de langue allemande. Il y a peu, l'historienne Ute Frevert considérait Frank comme représentatif de «l'utopie totalitaire d'un paternalisme étatique omniprésent». C'est face à une menace et des scénarios d'effondrement que l'interventionnisme fonctionne le mieux. Et il n'est pas dit que les politiques de prévention empêchent d'imposer des mesures se révélant problématiques par la suite.

Les articles publiés pour le $200^{\mathrm{e}}$ de la mort de Johann Peter Frank reflètent la variété de points de vue à propos de la prévention. Au sujet de ce "pionnier de la médecine préventive», l'Ärzteblatt Baden-Württemberg de juin 2021 écrit: «L'impératif de préserver la santé de la population en tant que garante de la solidité de l'Etat prenait pour lui le pas sur le droit et les aspirations de l'individu à la liberté. Comment s'étonner dès lors que le national-socialisme ait plus tard repris ses théories?»

En mars, deux spécialistes ont été conviés par l'Académie de santé publique de Düsseldorf pour débattre de la pertinence des écrits de Frank dans le contexte de l'actuelle pandémie. Ils ont discuté du bien-fondé de ses «strictes consignes d'hygiène», de l'importance d'un «Etat fort» et de la nécessité de disposer de «directives claires». Aucun mot en revanche sur le dilemme fondamental inhérent aux idées de Frank et l'idée même de prévention.

En temps de crise, il serait judicieux de ne pas perdre de vue ce dilemme ambivalent. A l'exemple de Johann Peter Frank, l'histoire de la médecine est parfois salutaire, rappelant les possibles effets indésirables des politiques de prévention.

Pour les références bibliographiques, merci de vous adresser à l'auteur. 